\title{
Identification and characterization of two ammonium transporter genes in flowering Chinese cabbage (Brassica campestris)
}

\author{
Yunna Zhu, Yanwei Hao, Houcheng Liu, Guangwen Sun, Riyuan Chen*, \\ Shiwei Song** \\ College of Horticulture, South China Agricultural University, 510642, Guangzhou, People's Republic of China \\ *E-mail: rychen@scau.edu.cn Tel \& Fax: +86-20-38294595 \\ **E-mail: swsong@scau.edu.cn Tel \& Fax: +86-20-38294595
}

Received December 1, 2017; accepted February 2, 2018 (Edited by N. Sasaki)

\begin{abstract}
Ammonium transporters (AMTs), which include AMT1 and AMT2 subfamilies, have been identified and partially characterized in many plants. In this study, two AMT2-type genes from Brassica campestris, namely BcAMT2 and BcAMT2like, were identified and characterized. BcAMT2 and BcAMT2like are $2666 \mathrm{bp}$ and $2952 \mathrm{bp}$, encode proteins of 490 and 489 amino acids, respectively, and contain five exons and four introns. Transient expression of these proteins labelled with green fluorescence protein in onion epidermal cells indicated that both are located on the plasma membrane. When expressing BcAMT2 or BcAMT2like, the mutant yeast strain $31019 \mathrm{~b}$ could grow on medium containing $2 \mathrm{mM}$ ammonium as the only nitrogen source when expressing BcAMT2 or BcAMT2like, indicating that both are functional AMT genes. Quantitative PCR results showed that BcAMT2 and BcAMT2like were expressed in all tissues, but they displayed different expression patterns in the reproductive stages. BcAMT2s transcript levels in leaves were positively correlated with ammonium concentration and external $\mathrm{pH}$. Moreover, the expression BcAMT2s responded to diurnal change. Furthermore, the uncharged form of ammonium, i.e., ammonia, might also be transported by BcAMT2s. These results provide new insights into the molecular mechanisms underlying ammonium absorption and transportation by the AMT2 subfamily in $B$. campestris.
\end{abstract}

Key words: ammonium transporter, Brassica campestris, functional characterization, gene expression, nitrogen regime.

\section{Introduction}

Ammonium, as a major inorganic nitrogen $(\mathrm{N})$ source for plants, plays important roles in their growth and development. It triggers multiple physiological and morphological responses (Liu and von Wirén 2017). Mild ammonium increases resistance against pathogen infection by enhancing the $\mathrm{H}_{2} \mathrm{O}_{2}$ burst in leaves (Fernández-Crespo et al. 2015), improves drought tolerance by enhancing drought-induced abscisic acid accumulation (Ding et al. 2016), and increases phosphorus (Jing et al. 2012; Zeng et al. 2012) and iron (Zou et al. 2001) uptake involved in proton release and rhizosphere acidification. In addition, ammonium changes root system architecture by inhibiting root elongation and by stimulating lateral root branching and root hair growth (Bloom et al. 2003; Lima et al. 2010; Yang et al. 2011). Thus, ammonium is a preferable $\mathrm{N}$ source (Gazzarrini et al. 1999; Hachiya and Sakakibara 2017), especially under the predicted rise in carbon dioxide concentration (Hachiya and Sakakibara 2017). However, excessive ammonium is toxic to plants (Hao et al. 2016). Accordingly, a strictly regulated ammonium uptake system must exist, including abundant ammonium transporters and effective mechanisms regulating their activity (Song et al. 2011).

Membrane transportation and distribution of ammonium are mediated by ammonium transporters (AMTs) of the ammonium transporter/methylamine permease/rhesus family, which has been found in all domains of life (McDonald et al. 2012). The ammonium transport mediated by members of this family provides sufficient $\mathrm{N}$ for optimal growth in plants and microorganisms. Molecular and biophysical studies have suggested that there are two transport systems for ammonium uptake, the high-affinity and the low-affinity transport system, which are responsible for the biphasic influx of ammonium. The former system takes up ammonium at low external concentrations (micromolar level) (Camañes et al. 2009; Gazzarrini et al. 1999; Yuan 
et al. 2007), while the latter assures the maintenance of a large ammonium influx at high external concentrations (millimolar level) (von Wirén et al. 2000a). Working together, these two systems ensure the optimization of ammonium uptake from the soil by the roots.

According to their phylogenetic relationships, plant AMTs can be clustered into two subfamilies: AMT1 and AMT2 (Loqué and von Wirén, 2004; McDonald et al. 2012). Members of both subfamilies differ in several characteristics (Giehl et al. 2017; Li et al. 2016; McDonald et al. 2012; Neuhäuser et al. 2009; von Wittgenstein et al. 2014). First, gene structure and protein characteristics are more complex in AMT2 than in AMT1, as AMT2-type genes contain several introns (Castro-Rodríguez et al. 2016), whereas AMT1-type genes include no introns (Becker et al. 2002; von Wirén et al. 2000b; Yuan et al. 2007), except for AMT1;2 from Lotus japonicus (Salvemini et al. 2001). Phylogenetic analyses revealed that AMT2 is more closely related to the AMT of prokaryotes than to plant AMT1s (Loqué and von Wirén 2004; McDonald et al. 2012). In addition, there is only 20 to $25 \%$ identity among AMT2 families, while plant AMT1s share 65 to $95 \%$ identity (Pantoja 2012). Second, in addition to mediating ammonium transport, AMT2;1 mediates electroneutral ammonia transport (Guether et al. 2009; Neuhäuser et al. 2009; Sohlenkamp et al. 2002). Thus, theoretically, AMT2;1 transports ammonium from the cytosol into the apoplast more efficiently than AMT1-type transporters (Giehl et al. 2017). Third, AMT1-type subfamily members play important roles in high-affinity ammonium uptake in the micromolar range (McDonald et al. 2012; Song et al. 2017; Yuan et al. 2007), while AMT2 subfamily members have a limited contribution to low-affinity ammonium in the millimolar range (Giehl et al. 2017). The ammonium transport capacity of AMT2;1 appears to increase with pH (Neuhäuser et al. 2009; Sohlenkamp et al. 2002). Finally, AMT2 subfamily members mainly translocate ammonium from root to shoot in Arabidopsis thaliana (Giehl et al. 2017). Although AMT2;1 in Arabidopsis is a functional ammonium transporter (Neuhäuser et al. 2009; Sohlenkamp et al. 2002), members of the AMT2 subfamily from different species typically exhibit different characteristics and appear to have different physiological roles and transport mechanisms ( $\mathrm{Li}$ et al. 2016). Therefore, the isolation, expression analysis, and functional analysis of new AMT2 subfamily members would be beneficial for gaining an understanding of the physiological roles of AMT2-type genes in plant growth.

Flowering Chinese cabbage (Brassica campestris L. ssp. chinensis var. utilis Tsen et Lee), a prominent vegetable in South China, has the largest growing area and yield in several locations. The flower stalk, the edible organ of flowering Chinese cabbage, is crisp and nutrientrich (Song et al. 2012). In a previous study, we found that fertilizers containing both nitrate and ammonium were more beneficial to flowering Chinese cabbage qualities than those with a single $\mathrm{N}$ source (Song et al. 2012). Nitrate may stimulate ammonium loading into the xylem and/or its assimilation through the enhanced amino acid biosynthesis (Hachiya and Sakakibara 2017). In A. thaliana, ammonium is imported into the cytosol by AtAMT1;1 and AtAMT2;1, which are expressed in the leaves, or via other pathways through diffusion or by unknown transporters/channels (Ludewig et al. 2007). In addition, AMT2;1 plays an important role in rootto-shoot translocation of ammonium in Arabidopsis (Giehl et al. 2017). However, there is no information regarding ammonium transport systems and AMT2 subfamily roles in $B$. campestris. Therefore, research on ammonium transport systems in $B$. campestris is very important for gaining an understanding of the regulation of ammonium uptake by the roots. In the present study, two AMT2-type genes were isolated from B. campestris and their expression patterns were analysed to provide information on the AMT family and discuss the possible roles of these two AMT2s in this species.

\section{Materials and methods}

\section{Plant materials and culture conditions}

The present study examined the flowering Chinese cabbage variety "Youlv 501" (Guangzhou Academy of Agriculture Sciences, Guangdong province, China). Experiments were carried out in a greenhouse $\left(25\right.$ to $30^{\circ} \mathrm{C}$, natural light). Seeds were surface-sterilized in $2.5 \% \mathrm{NaClO}$ for $10 \mathrm{~min}$, washed with distilled water, and sown in plug trays filled with perlite. Plug seedlings were grown until the third true leaf was fully expanded. Then, 16 uniform seedlings were transplanted to a plastic box $(60 \mathrm{~cm}$ long $\times 40 \mathrm{~cm}$ wide $\times 15 \mathrm{~cm}$ high $)$ filled with 241 of modified Hoagland solution $\left(4.0 \mathrm{mM} \mathrm{NaNO}_{3}, 1.0 \mathrm{mM}\right.$ $\mathrm{KH}_{2} \mathrm{PO}_{4}, 2.0 \mathrm{mM} \mathrm{KCl}, 1.0 \mathrm{mM} \mathrm{MgSO}, 0.5 \mathrm{mM} \mathrm{CaCl}_{2}, 0.1 \mathrm{mM}$ Fe-EDTA, $50 \mu \mathrm{M} \mathrm{H}_{3} \mathrm{BO}_{3}, 12 \mu \mathrm{M} \mathrm{MnSO}_{4}, 1 \mu \mathrm{M} \mathrm{ZnCl}_{2}, 1 \mu \mathrm{M}$ $\mathrm{CuSO}_{4}$, and $\left.0.2 \mu \mathrm{M} \mathrm{Na} \mathrm{MoO}_{4}\right)$. The nutrient solution was completely replaced every four days and its $\mathrm{pH}$ was adjusted to 5.8 every two days. An air pump was installed at $15 \mathrm{~min} \mathrm{~h}^{-1}$ to maintain good air circulation. Roots, stems, leaves and other organs were randomly selected and harvested separately at the following growth stages: roots $(\mathrm{R})$, hypocotyls $(\mathrm{H})$, and cotyledons $(\mathrm{C})$ were harvested at cotyledon stage (stage 1); roots $(\mathrm{R})$, stem $(\mathrm{S})$, and leaves $(\mathrm{L})$ were harvested at the threeleaf stage (stage 2) and six-leaf stage (stage 3); roots (R), stem $(\mathrm{S})$, leaves (L), and flower buds (B) were harvested at the stalk-growth stage (stage 4); roots (R), stem (S), leaves (L), flowers $(\mathrm{F})$, and pods $(\mathrm{P})$ were harvested at the flowering stage (stage 5). All samples were randomly selected four plants per biological replicate and harvested separately during the several growth stages.

After 10 to 15 days in nutrient solution, the seedlings were used in experiments. For $\mathrm{N}$-starvation and $\mathrm{N}$-resupply 
treatments, seedlings were washed in deionized water and transferred to $\mathrm{N}$-free modified Hoagland solution containing $4 \mathrm{mM} \mathrm{NaCl}$ instead of $4 \mathrm{mM} \mathrm{NaNO}_{3}$ for $72 \mathrm{~h}$. After this period, the nutrient solution was replaced with a solution containing $4 \mathrm{mM} \mathrm{NH}_{4} \mathrm{Cl}$ as the only $\mathrm{N}$ source. Roots and leaves were harvested at $0,24,48$, and $72 \mathrm{~h}$ during the $\mathrm{N}$-deficiency treatment and at 2, 4, and $8 \mathrm{~h}$ during the $\mathrm{N}$-resupply treatment. For different $\mathrm{pH}$ treatments, seedlings were transferred to a modified Hoagland solution, where $4 \mathrm{mM} \mathrm{NH}_{4} \mathrm{Cl}$ replaced $4 \mathrm{mM} \mathrm{NaNO}_{3}$, and $\mathrm{pH}$ values were adjusted to $4.8,5.8$, and 6.8 with $2 \mathrm{M} \mathrm{HCl}$ or $\mathrm{NaOH}$. After three days, roots and leaves were harvested. For the high-ammonium treatment, seedlings were kept in a $10 \mathrm{mM} \mathrm{NH}_{4} \mathrm{Cl}$ solution, and roots and leaves were harvested at $0,24,48$, and $72 \mathrm{~h}$. For the circadian rhythm study, seedlings were cultured in a greenhouse $\left(25\right.$ to $30^{\circ} \mathrm{C}$, natural light), and then transferred to a growth incubator at $25^{\circ} \mathrm{C}, 70 \%$ relative humidity, $150 \mu \mathrm{mol} \mathrm{m} \mathrm{m}^{-2} \mathrm{~s}^{-1}$ light intensity, and a $12 / 12$ h light/dark period (light, 7:00 to 19:00; dark 19:00 to 7:00). After three days, leaves and roots were first sampled at 3:00 to 24:00 and then every $3 \mathrm{~h}$. All samples were immediately frozen in liquid nitrogen and stored at $-80^{\circ} \mathrm{C}$ for quantitative realtime PCR (qPCR).

\section{BcAMT2s gene cloning and bioinformatics analysis}

Based on AMT2 and AMT2like sequences of B. rapa (retrieved from GenBank, under accessions no. XM_009145156.2, XM_009143483.2, respectively) specific primers were designed for each gene (Table 1). Total RNA was extracted from leaves and roots of $B$. campestris using the Plant RNAiso Plus Kit (TaKaRa, Kyoto, Japan), and was reverse transcribed using the PrimeScript ${ }^{\mathrm{TM}} 1$ st Strand cDNA Synthesis Kit (TaKaRa). Genomic DNA was extracted from leaves using the plant genomic DNA kit (Tiangen Biotechnology, Beijing, China). Full-length sequences of the ORFs of the BcAMT2s were amplified by PCR using CDNA of B. campestris as template, and the products were ligated into the pYES2 vector
(Waryong Biotechnology, Beijing, China) and sequenced. The corresponding genomic sequences were obtained in a similar way, using genomic DNA as the PCR template.

According to the deduced amino acid sequence, the following protein characteristics were predicted using online tools: protein physicochemical properties (ProtParam, http:// web.expasy.org/protparam/), PROSITE motifs (ScanProsite, http://prosite.expasy.org/scanprosite/), transmembrane motifs (Protter, http://wlab.ethz.ch/protter/), and subcellular localization (http://www.softberry.com). Multiple sequence alignment of 38 AMT proteins from A. thaliana, B. rapa, B. campestris, Oryza sativa, P. trichocarpa, and Escherichia coli, and phylogenetic analysis based on an unrooted, maximum likelihood-based phylogenetic tree were conducted using CLUSTALW in MEGA (6.0) (Tamura et al. 2013). Exons/ introns in cDNA and genomic sequences were visualized in GSDS (2.0) (http://gsds.cbi.pku.edu.cn/) (Hu et al. 2015). Conserved domains defining the AMT subfamilies were generated using WEBLOGO (http://weblogo.berkeley.edu/logo. cgi/) (Crooks et al. 2004).

\section{Sub-cellular location}

Primers pB2 and pB2like (Table 1) were used to amplify the coding sequences of BcAMT2 and BcAMT2like. The amplicons were ligated into the pBI121-GFP vector (kindly provided by Dr. Guibing Hu, South China Agricultural University, China), which harbours the CaMV 35 S promoter and GFP, at the XbaI and SmaI sites, and the fusion constructs were transformed into onion epidermal cells by the Agrobacterium tumefaciens (EHA105; Weidi Biotechnology, Shanghai, China) infiltration method (Zhang et al. 2013). After two days of incubation, the fluorescence levels of the fusion proteins were examined under a positive fluorescence microscope (Axio Imager D2; Zeiss, Dresden, Germany) under $480 \mathrm{~nm}$ excitation and $525 \mathrm{~nm}$ emission wavelengths.

Table 1. Primers used in the experiments.

\begin{tabular}{|c|c|c|}
\hline Primer name & Sequence $\left(5^{\prime}-3^{\prime}\right)$ & Usage \\
\hline AMT2 & $\begin{array}{l}\text { F: CAGTGTGCTGGAATTCATGGCCGGCGCTTACGATGCGA } \\
\text { R: TGCGGCCCTCTAGATTATAAAACAATAGTGACACCTC }\end{array}$ & $\begin{array}{l}\text { Cloning } B c A M T 2 \text { and } B c A M T 2 l i k e \text { into pYES2 vector } \\
\text { using EcoRI and XbaI sites }\end{array}$ \\
\hline AMT2like & $\begin{array}{l}\text { F: CAGTGTGCTGGAATTCATGGCCGGAGCTTACGGTGCA } \\
\text { R: TGCGGCCCTCTAGATTATAAAACAATGGTAACACCTC }\end{array}$ & \\
\hline $\mathrm{pB} 2$ & $\begin{array}{l}\text { F:CACGGGGGACTCTAGAATGGCCGGCGCTTACGATGCGA } \\
\text { R: TCCTTTACCCATCCCGGGTAAAACAATAGTGACACCTC }\end{array}$ & $\begin{array}{l}\text { Cloning } B c A M T 2 \text { and } B c A M T 2 l i k e \text { into pBI121 vector } \\
\text { using XbaI and SmaI sites }\end{array}$ \\
\hline pB2like & $\begin{array}{l}\text { F: CACGGGGGACTCTAGAATGGCCGGAGCTTACGGTGCA } \\
\text { R: TCCTTTACCCATCCCGGGTAAAACAATGGTAACACCTC }\end{array}$ & \\
\hline qAMT2 & $\begin{array}{l}\text { F: CTGTCTGGAATGTAGTGTCG } \\
\text { R: TCTCCATCTCCCCAAAGAGC }\end{array}$ & qPCR \\
\hline qAMT2like & $\begin{array}{l}\text { F: AGCTTGGGATTGGAGATGACG } \\
\text { R: ATGCCTTGTGGCATCGAAC }\end{array}$ & qPCR \\
\hline$\beta$-actin & $\begin{array}{l}\text { F: GTGACAATGGAACTGGAATGG } \\
\text { R: AGACGGAGGATAGCGTGAGG }\end{array}$ & qPCR \\
\hline GAPDH & $\begin{array}{l}\text { F: CAGGTTTGGAATTGTCGAGG } \\
\text { R: GAGCTGTGGAAGCACCTTTC }\end{array}$ & $\mathrm{qPCR}$ \\
\hline
\end{tabular}




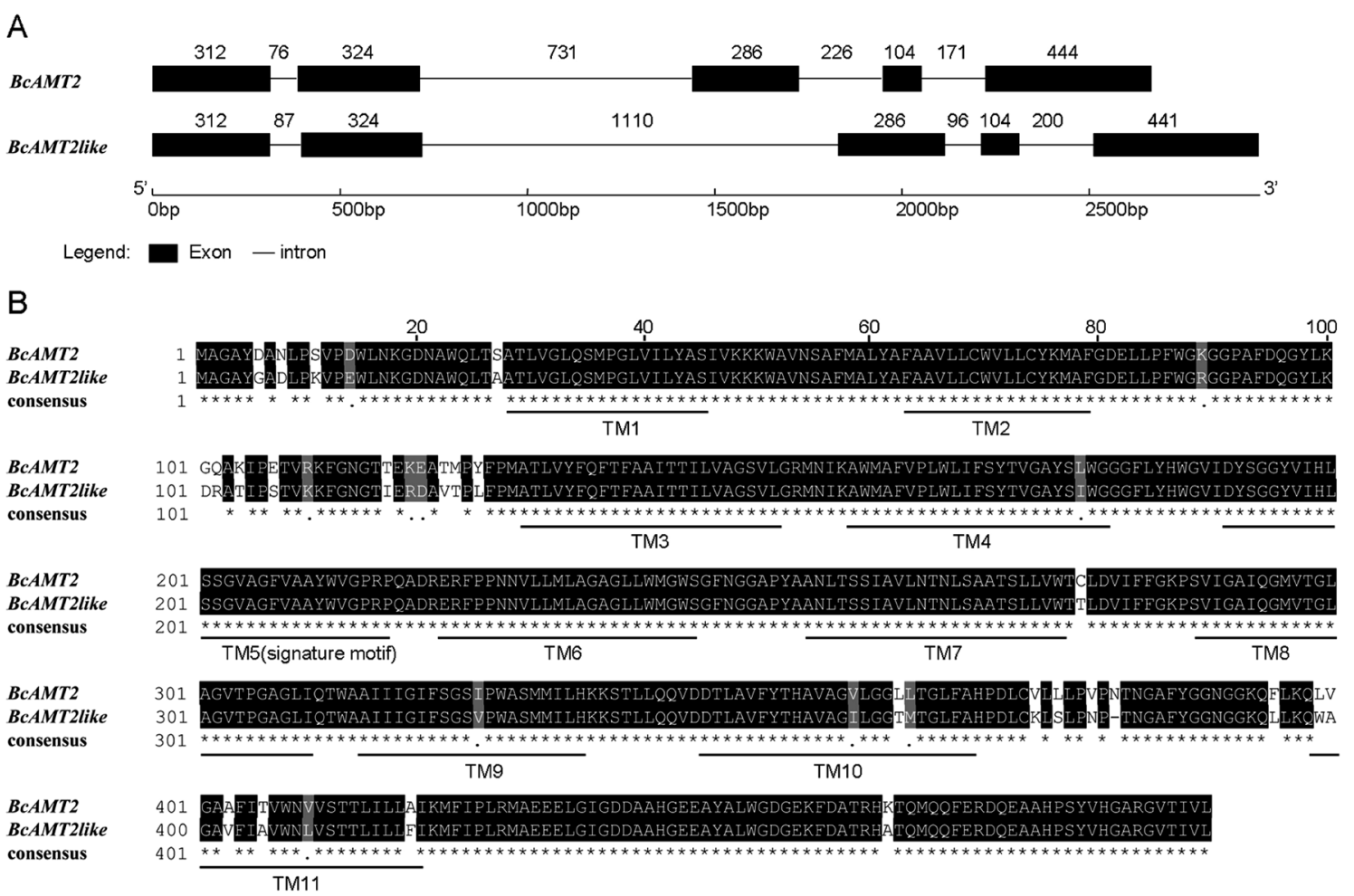

Figure 1. Genomic structure and multiple alignment of the ammonium transporters (AMT) BcAMT2 and BcAMT2like isolated from Brassica campestris. A: Gene structure analysis was carried out in GSDS (gene structure display server, http://gsds.cbi.pku.edu.cn/). Numbers above exons (boxes) and introns (lines) indicate the number of nucleotides. B: Multiple alignment was performed in MEGA 6.0. Transmembrane domains (TM) and AMT signature motifs were predicted by Protter (http://wlab.ethz.ch/protter/) and ExPaSy (http://expasy.org/tools/scanprosite/), respectively. Identical residues are indicated by asterisks, different residues are indicated as a gap, semi conservative mutations are shown as points, predicted TM and signature motifs are outlined by thick lines.

\section{$q P C R$}

Total RNA was extracted from samples using the Plant RNAiso Plus Kit (TaKaRa), and was reverse transcribed using the PrimeScript ${ }^{\mathrm{TM}}$ RT reagent Kit with gDNA Eraser (TaKaRa). qPCR was performed in a LightCycler 480 Real-Time PCR System (Roche, Basel, Switzerland), using SYBR Premix Ex Taq (TaKaRa) and the primer pairs listed in Table 1. The genes encoding glyceraldehyde-3-phosphate dehydrogenase and $\beta$-actin were used as internal control genes. The PCR mixture $(10 \mu \mathrm{l}$ total volume) contained $5 \mu \mathrm{l} 2 \times$ SYBR Premix Ex Taq, $0.4 \mu \mathrm{l}$ each primer $(10 \mu \mathrm{M}), 2 \mu \mathrm{l} 10$-fold diluted cDNA,

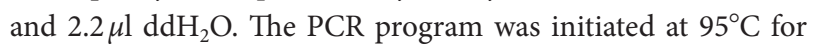
$30 \mathrm{~s}$, followed by 45 cycles of $95^{\circ} \mathrm{C}$ for $5 \mathrm{~s}$ and $60^{\circ} \mathrm{C}$ for $30 \mathrm{~s}$, and completed with a melting curve analysis $\left(65\right.$ to $95^{\circ} \mathrm{C}$, at increments of $0.5^{\circ} \mathrm{C}$ ), which was conducted to confirm primer specificity. No-template (blank) controls were included in every reaction batch. Three biological replicates were used to calculate relative gene expression as previously described (Livak and Schmittgen 2001).

\section{Yeast growth and complementation}

Yeast expression vectors were constructed by cloning the $B c A M T 2$ and BcAMT2like ORFs into the EcoRI and XbaI sites of the pYES2 vector (Waryong Biotechnology), respectively. The yeast mutant strain 31019b ( $\Delta m e p 1, \Delta m e p 2, \Delta m e p 3$, and ura3), which was kindly provided by Dr. Bruno André (Université Libre de Bruxelles, Belgium), is unable to grow on medium containing an ammonium concentration lower than $5 \mathrm{mM}$ as the only $\mathrm{N}$ source (Marini et al. 1997; Yuan et al. 2007). Recombinant or empty (pYES2) plasmids were transformed into $31019 \mathrm{~b}$ yeast cells using LiAcO (Yuan et al. 2007). Transformed cells were plated on synthetic dropout medium lacking uracil (FunGenome Company, Beijing, China) for the identification of positive clones. Positive clones were pre-cultured in liquid yeast nitrogen base medium without amino acids, and ammonium sulphate was supplemented until the optical density at $600 \mathrm{~nm}$ reached 1.0. Growth complementation assays were performed on solid yeast nitrogen base medium at $\mathrm{pH} 5.8$ containing $2 \%$ galactose and a single $\mathrm{N}$ source $\left(1 \mathrm{mM}\right.$ arginine or $\left.2 \mathrm{mM} \mathrm{NH}_{4} \mathrm{Cl}\right)$. Overnight cultures were serially diluted by factors of 10 , and $3 \mu \mathrm{l}$ of precultured yeast cell suspensions at each dilution were spotted on yeast nitrogen base medium. Growth was evaluated after three days of incubation at $30^{\circ} \mathrm{C}$.

\section{Statistical analysis}

Data from three independent experiments were analysed by one-way analysis of variance using SigmaPlot (11.1) (Jandel Scientific software, San Rafael, CA, USA), and differences were compared using Duncan's test considering $p<0.05$ as the significance threshold. 


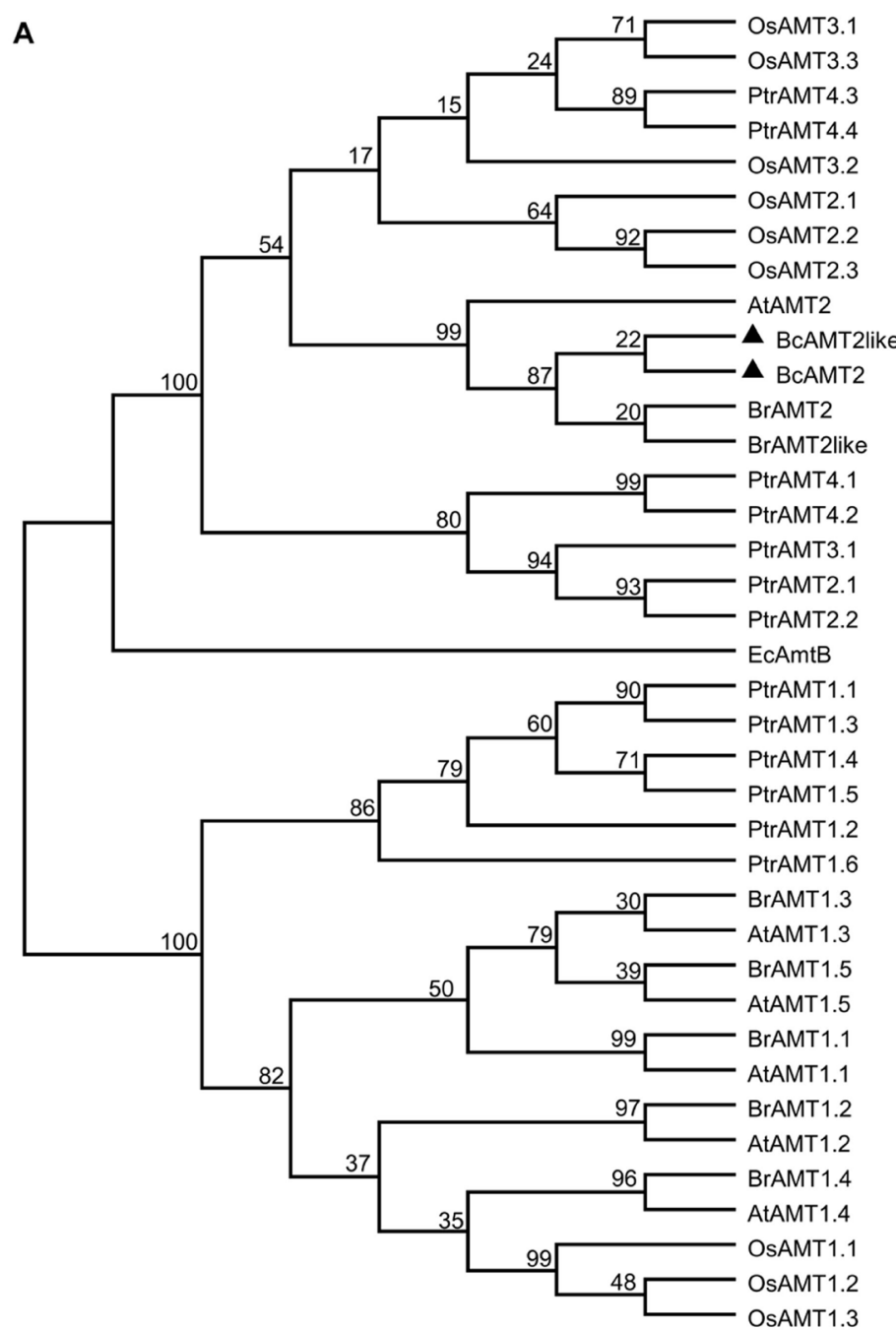

Subfamily 2

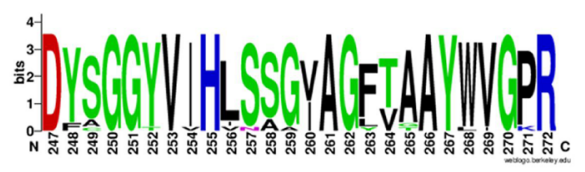

Subfamily 1

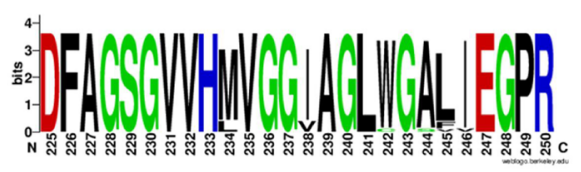

Figure 2. Phylogenetic analysis and conserved domains of ammonium transporters (AMTs). A: Unrooted, maximum likelihood-based phylogenetic tree of AMT family proteins generated in MEGA 6.0 software, using ClustalW for the alignment and 1000 bootstrap replicates. AtAMT1;1-1;5 and AtAMT2 from A. thaliana (accessions no. NM_117425.2, NM_105152.3, NM_113336.3, NM_119012, NM_113335, and NM_129385.5); BrAMT1.1BrAMT1;5, BrAMT2, and BrAMT2like from B. rapa (XM_009126366.2, XM_009113156.1, XM_009104274.2, XM_009131146.2, XM_009137637.1, XM_009145156.2, and XM_009143483.2); OsAMT1;1-1;3, OsAMT2;1-2;3, OsAMT3;1-3;3 from O. sativa (NM_001059815.1, NM_001053990.1, NM_001053991.1, XM_009378133.2, XM_015787532.1, XM_015766098.1, XM_015766529.1, NM_001058371.2, and NM_001053632.1); PtrAMT1;1-1;5, PtrAMT2;1, PtrAMT2;2, PtrAMT3;1, PtrAMT4;1-T4;4 from P. trichocarpa (XM_002314482.2, XM_002325754.1, XM_002311667.2, XM_002303068.1, XM_002301801.1, XM_002314070.2, XM_002309115.1, XM_002323564.1, XM_002300186.2, XM_002302048.2, XM_002324235.2, XM_002306767.2, and XM_002319034.2); EcAmtB from E. coli (NP_286193); BcAMT2 and BcAMT2like from B. campestris are displayed with a black triangle. B: Conserved domains defining the AMT subfamilies generated using WEBLOGO (http://weblogo.berkeley.edu/logo.cgi/).

\section{Results}

\section{Gene isolation and sequence analysis}

Two AMT2-type homologous genes, namely BcAMT2 and BcAMT2like (accession no. MF966941 and MF966942), were isolated from genomic DNA of $B$. campestris. Although both genes had the same number of introns and exons, the lengths thereof differed (Figure 1A). The complete open reading frame (ORF) of BcAMT2 comprised 1470 nucleotides and encoded a $52.63 \mathrm{kD}$ polypeptide of 490 amino acids. The DNA sequence of BCAMT2 was composed of 2666 nucleotides (accession no. MF966943) and it included five exons of 104 to $444 \mathrm{bp}$ and four introns of 76 to $731 \mathrm{bp}$ (Figure
1A). BcAMT2like encoded a $52.53 \mathrm{kD}$ polypeptide of 489 amino acids, and it contained 2952 nucleotides distributed in five exons separated by four introns (accession no. MF966944) (Figure 1A).

At the amino acid level, the similarity of BcAMT2 and BcAMT2like was 93\% (Figure 1B). Based on their predicted structures, both BcAMT2 and BcAMT2like are located on the plasma membrane and have 11 transmembrane (TM) domains (Figure 1B), an extracellular $\mathrm{N}$-terminus, and a cytosolic C-terminus. The predicted signature motif of both BcAMT2 and BcAMT2like is ${ }^{191}$ DYSGGYVIHLSSGVAGFVAAYWVGPR ${ }^{216}$, but the proteins differ in one, three, and five amino acid residues 


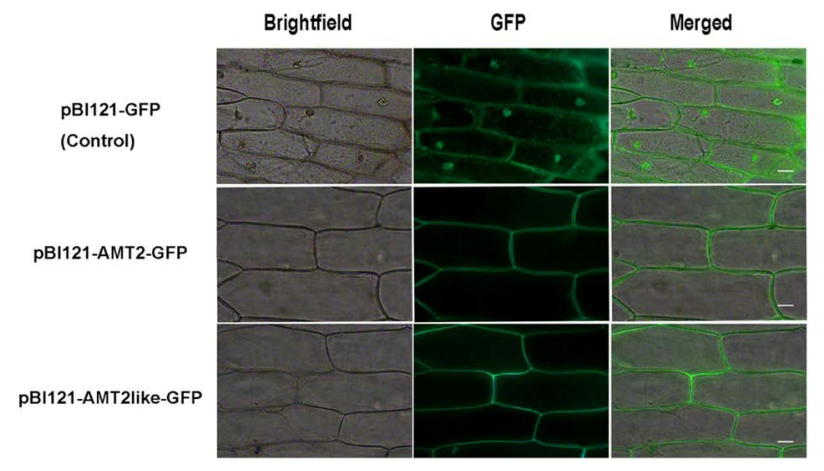

Figure 3. Subcellular localization of pBI121-GFP, pBI121-BcAMT2GFP, and pBI121-BcAMT2like-GFP fusion proteins in onion epidermal cells. Bright field: image obtained by bright field microscopy; GFP: green fluorescence derived from GFP imaged by fluorescence microscopy; Merged: overlay of the two images. Scale bar: $50 \mu \mathrm{m}$.

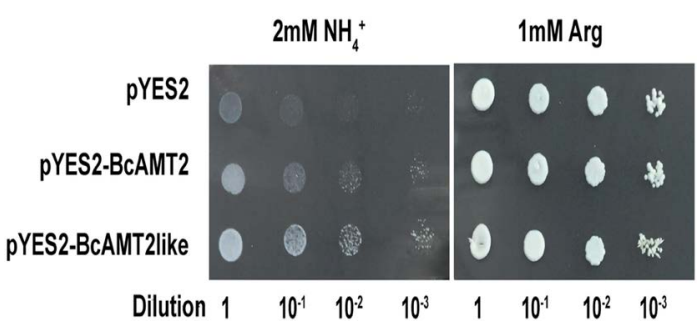

Figure 4. Functional complementation of yeast mutant $31019 \mathrm{~b}$ cells by $B c A M T 2$ and BcAMT2like. pYES2: empty vector was used as the negative control. pYES2-BcAMT2 and pYES2-BcAMT2like: BcAMT2 and BcAMT2like ORFs cloned into pYES2 vector. Yeast cell suspensions were adapted to an optical density at $600 \mathrm{~nm}$ of 1.0 (dilution 1). Then, they were serially diluted by factors of 10 , and $3 \mu \mathrm{l}$ of the yeast cell suspensions at each dilution were spotted on yeast nitrogen base medium. Growth was evaluated after three days of incubation at $30^{\circ} \mathrm{C}$.

in TM 9th, 10th, and 11th, respectively (Figure 1B).

Phylogenetic analysis based on multiple sequence alignment of 38 AMT proteins from six species, as retrieved from GenBank or obtained in the present study, showed that AMTs were divided into two subfamilies (Figure 2A). Both BcAMT2 and BcAMT2like, which were grouped within the AMT2 subfamily, were closely related to EcamtB from Escherichia coli and most related to BrAMT2 from B. rapa, and were classified into the same cluster as AtAMT2 from A. thaliana. However, PtrAMT2s from Populus trichocarpa were classified into a different cluster (Figure 2A).

Each subfamily was defined by a conserved domain: in the AMT1 subfamily, this domain spanned residues $\mathrm{D}^{225}$ to $\mathrm{R}^{250}$ while in AMT2 subfamily, it spanned $\mathrm{D}^{247}$ to $\mathrm{R}^{272}$ (Figure 2B, Crooks et al. 2004).

The observation of fluorescence in onion cells transiently transfected with recombinant plasmids including full-length sequences of BcAMT2 and BcAMT2like fused with green fluorescent protein (GFP) confirmed that both proteins were located within the plasma membrane (Figure 3).

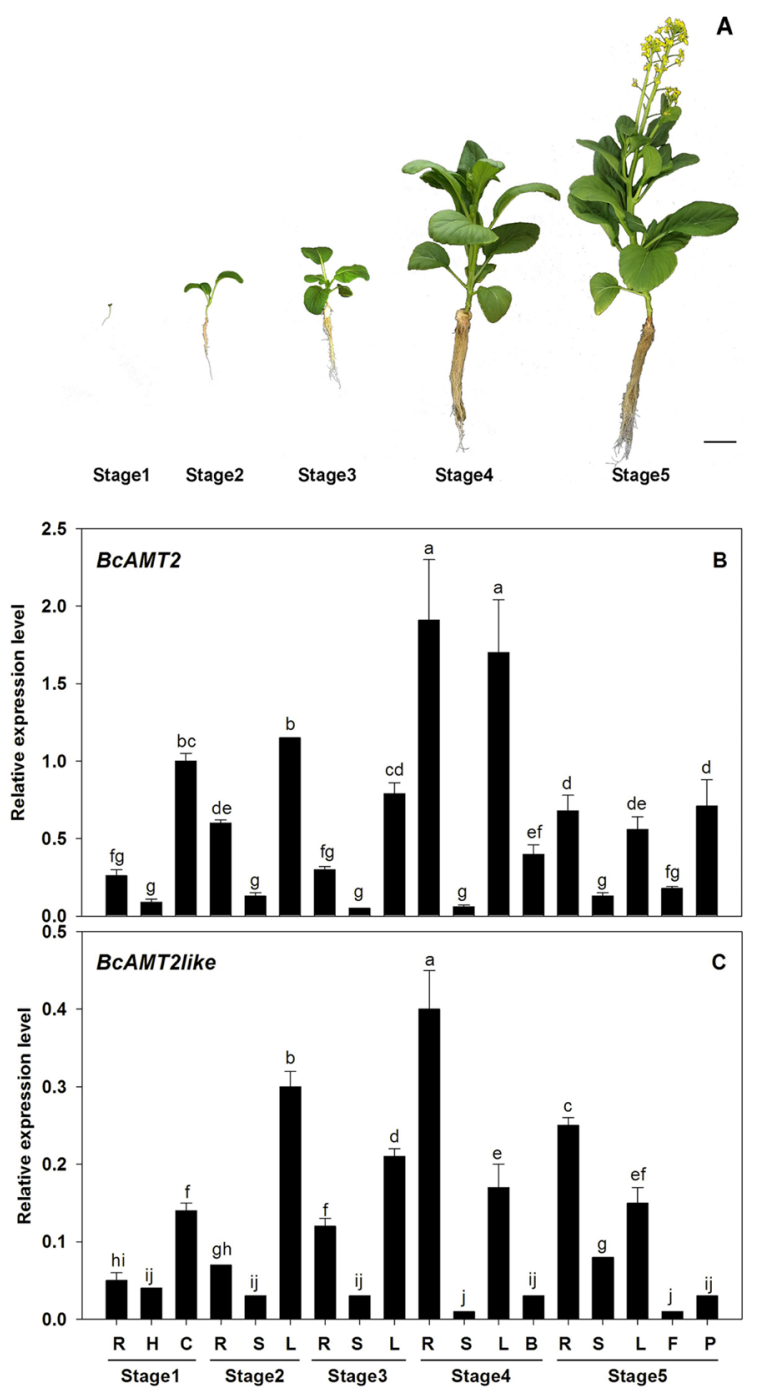

Figure 5. BcAMT2 and BcAMT2like expression patterns in different organs of B. campestris during five developmental stages. A: Samples were collected at the cotyledon stage (stage 1), three-leaf stage (stage 2), six-leaf stage (stage 3), stalk-growth stage (stage 4), and flowering stage (stage 5). Scale bar: $5 \mathrm{~cm}$. B, C: Expression levels of BcAMT2 and BcAMT2like in the different stages. Roots (R), hypocotyls $(\mathrm{H})$, cotyledons (C), stem (S), leaves (L), buds (flower bud), flowers (F), and pods (P). The transcript levels of BcAMT2 and BcAMT2like were normalized to the expression of $\beta$-actin and glyceraldehyde-3-phosphate dehydrogenase gene. Each value represents the mean \pm SE $(n=3)$. Different lowercase letters show significant differences at $p<0.05$.

\section{Functional expression of BCAMT2 and BcAMT2like in yeast}

Recombinant yeast strains harbouring pYES2-BcAMT2 or pYES2-BcAMT2like or the empty vector (pYES2) grew normally on solid medium containing $1 \mathrm{mM}$ arginine, suggesting that vector construction and yeast-cell growth abilities were adequate (Figure 4). Recombinant strains harbouring pYES2-BcAMT2 and pYES2-BcAMT2like grew normally on solid medium with $2 \mathrm{mM}$ ammonium as the only $\mathrm{N}$ source, while negative control cells transformed with empty pYES2 could hardly grow or did not grow at all (Figure 4). These 
results indicated that $B c A M T 2$ and $B c A M T 2$ like encode functional AMTs.

\section{Expression patterns of BcAMT2s in different tissues and developmental stages}

To further characterize the AMT2 subfamily in $B$. campestris, the expression patterns of BcAMT2 and $B c A M T 2$ like in different organs and developmental stages were investigated. As shown in Figure 5, BcAMT2 was constitutively expressed throughout the growth period and in all tested organs, showing the highest expression in cotyledons and leaves during vegetative growth and the lowest in stems throughout development (Figure $5 \mathrm{~B}$, stages 1 to 3 ). In addition, the expression level of $B c A M T 2$ in roots and pods gradually increased during the stages of reproductive growth (Figure 5B, stages 4 and 5). Similar trends were observed in BcAMT2like expression levels from the cotyledon stage to stalkgrowth stage (Figure 5C, stages 1 to 3 ), but expression levels in roots and stems significantly increased during reproductive growth stages (Figure 5C, stages 4 and $5)$. Thus, there was an obvious difference between the expression of BcAMT2 and BcAMT2like during the reproductive growth stage, with high $B c A M T 2$ expression in roots, leaves, flower buds, and pods (Figure 5B) and high BcAMT2like expression in roots, leaves, and stem only (Figure 5C). This indicated that BcAMT2 and $B c A M T 2$ like might play different roles during the late development of $B$. campestris.

\section{BcAMT2s transcriptions respond to $N$ regimes, external $\mathrm{pH}$, and circadian cycle}

The results of $\mathrm{qPCR}$ showed that BcAMT2 and $B c A M T 2$ like had similar expression patterns in leaves during $\mathrm{N}$ starvation and resupply (Figure 6A, B). With the prolongation of $\mathrm{N}$ starvation, the transcription of both genes strongly decreased, with BcAMT2 and BcAMT2like expression being about $15.8 \%$ and $24.9 \%$ of that under control conditions, respectively. During $\mathrm{N}$ resupply, their expression levels increased gradually. In roots, BcAMT2 and BcAMT2like had different expression patterns (Figure 6C, D). The expression of both genes slightly decreased as compared to that under control conditions under $\mathrm{N}$ starvation; however, $4 \mathrm{~h}$ after $\mathrm{N}$ resupply, only BcAMT2like transcripts increased.

When $B$. campestris plants were exposed to a high ammonium concentration $(10 \mathrm{mM}), B c A M T 2$ and $B c A M T 2$ like expression strongly increased in leaves (Figure 7A, B), but not in roots, where levels were nearly unaffected (Figure $7 \mathrm{C}$ ), except for the decline in BcAMT2like expression at $24 \mathrm{~h}$ after ammonium supply (Figure 7D). Given that $10 \mathrm{mM}$ ammonium exceeds
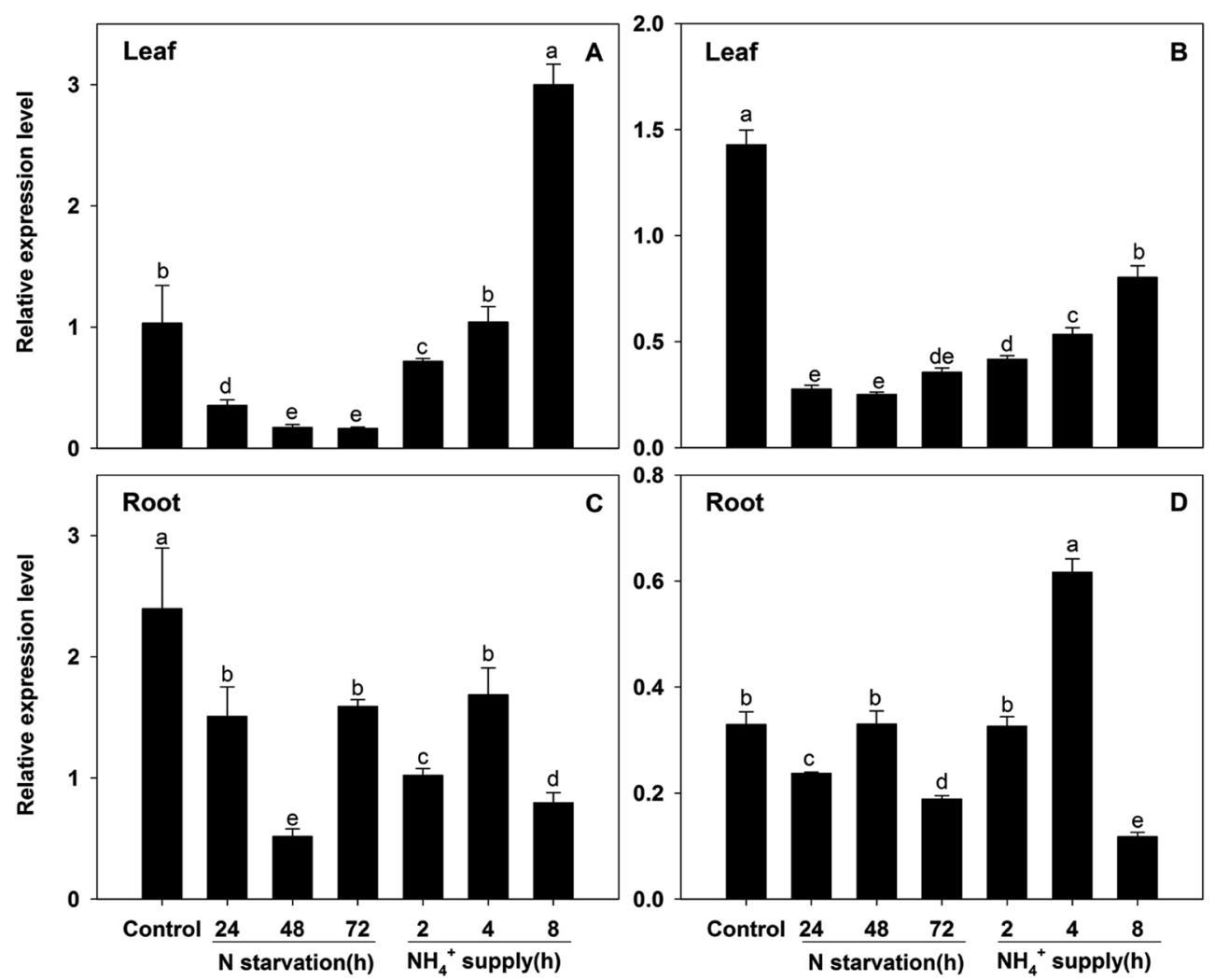

Figure 6. BcAMT2 and BcAMT2like expression in B. campestris after different periods of $\mathrm{N}$ starvation and ammonium $\left(\mathrm{NH}_{4}^{+}\right)$supply. A, B: BcAMT2 and BcAMT2like expression in leaves under $\mathrm{N}$ starvation for $72 \mathrm{~h}$, followed by $\mathrm{NH}_{4}^{+}$supply for $8 \mathrm{~h}$. C, D: BcAMT2 and BcAMT2like expression in roots under the same conditions described for leaves. Each value represents the mean $\pm \mathrm{SE}(n=3)$. Different lowercase letters indicate significant differences at $p<0.05$. 

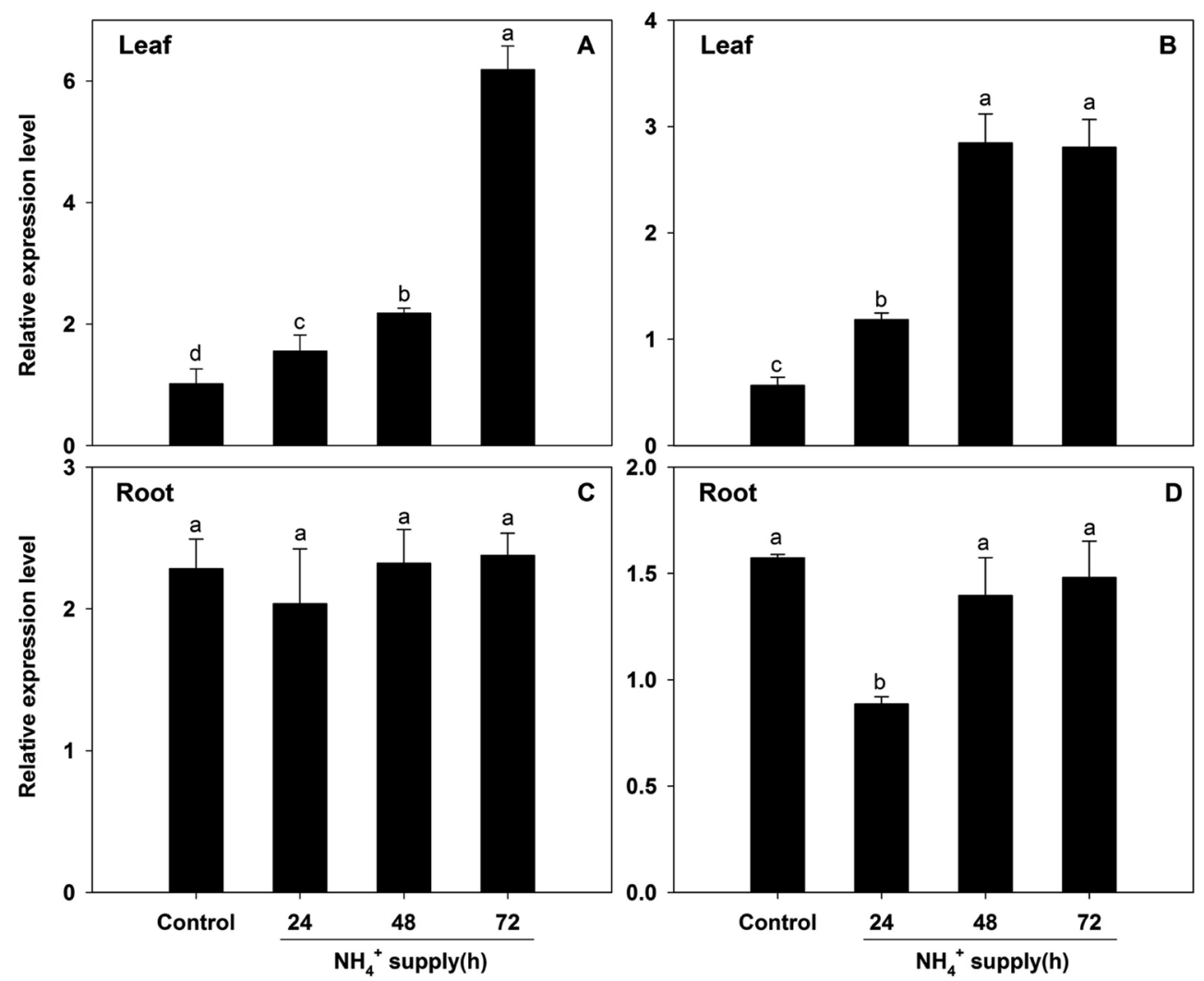

Figure 7. BcAMT2 and BcAMT2like expression in leaves and roots of $B$. campestris under high $\mathrm{NH}_{4}^{+}$treatment (10 mM). A, B: BcAMT2 and BcAMT2like expression in leaves treated for the indicated periods; C, D: BcAMT2 and BcAMT2like expression in roots treated for the indicated periods. Each value represents the mean \pm SE $(n=3)$. Different lowercase letters indicate significant differences at $p<0.05$.
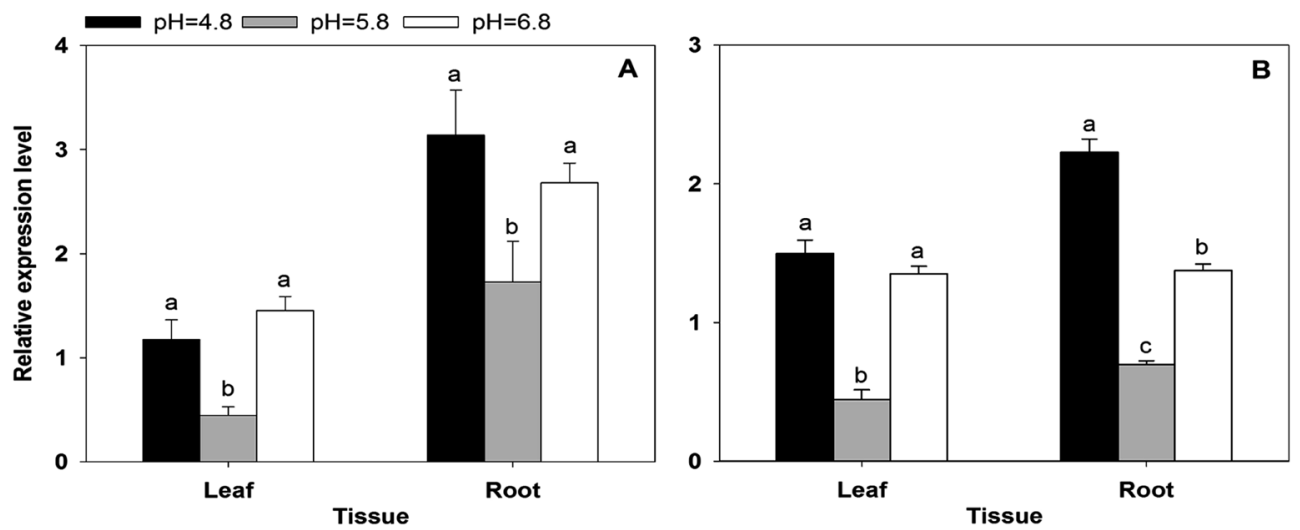

Figure 8. BcAMT2 (A) and BcAMT2like (B) expression in leaves and roots of B. campestris at different $\mathrm{pH}$ for three days. Each value represents the mean \pm SE $(n=3)$. Different lowercase letters indicate significant differences at $p<0.05$.

the root assimilatory capacity, plants might require enhanced long-distance transport and xylem loading of excess ammonium to prevent the deleterious effects of ammonium accumulation in roots. The observed expression patterns indicated that these two genes might act in root-to-shoot transport and are likely regulated by the levels of ammonium within the plant.

Environmental $\mathrm{pH}$ has a significant influence on the balance between ammonium and ammonia, and the expression levels of BcAMT2 and BcAMT2like in
B. campestris exhibited strong responses to external $\mathrm{pH}$ (Figure 8). When the $\mathrm{pH}$ increased from 4.8 to 5.8, transcript levels of $B c A M T 2$ in leaves and roots decreased by $62 \%$ and $45 \%$ in relation to those in the control treatment, respectively, and returned to the original levels when the $\mathrm{pH}$ increased from 5.8 to 6.8 (Figure 8A). Similar results were obtained for BcAMT2like (Figure $8 \mathrm{~B})$, suggesting that the responses of both genes to ammonium might be $\mathrm{pH}$-dependent.

Circadian variations (Figure 9) were detected in 


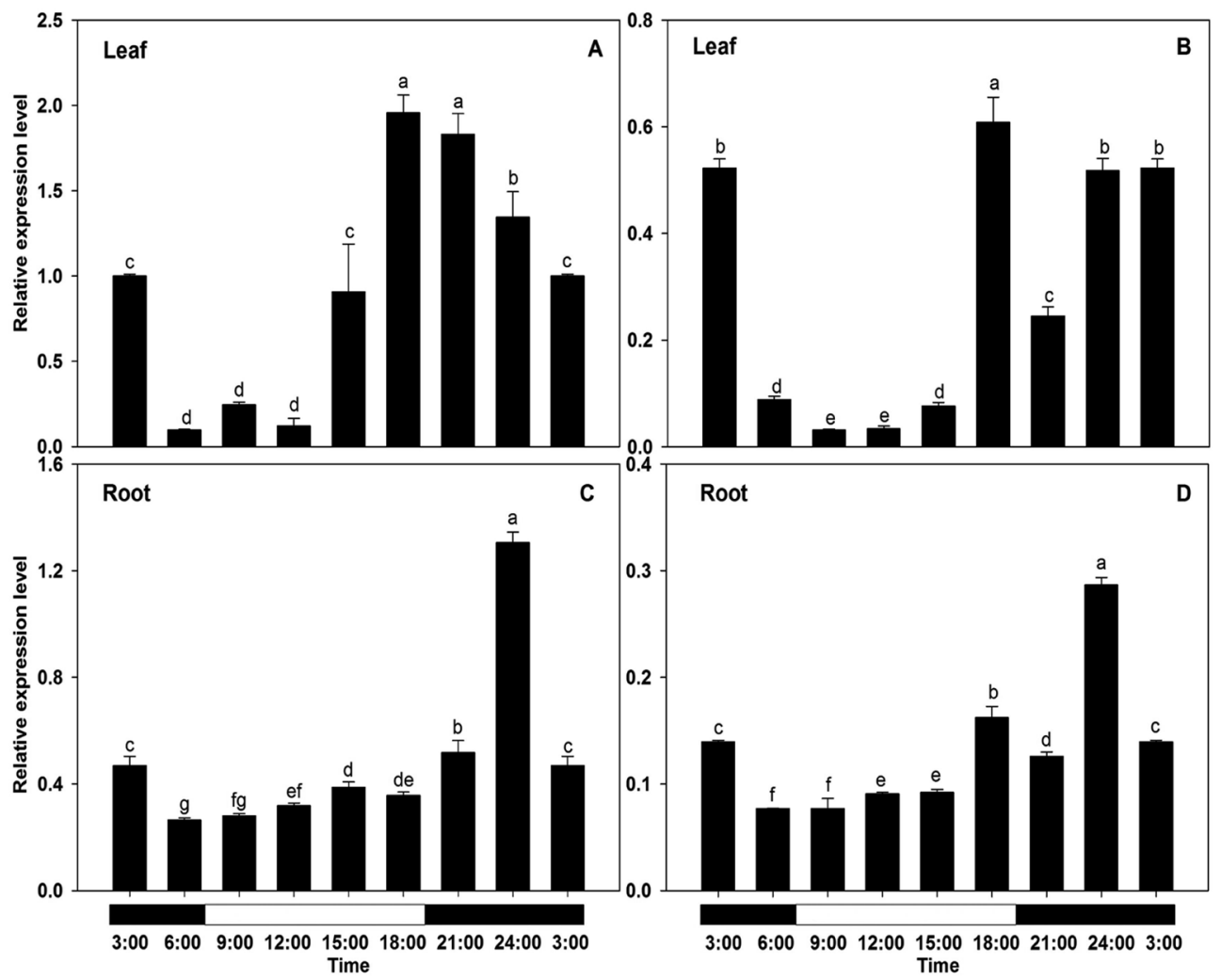

Figure 9. Effect of the circadian cycle on the expression of BcAMT2 and BcAMT2like in Brassica campestris. A, B: expression of BcAMT2 and $B c A M T 2$ like in leaves; C, D: expression of BcAMT2 and BcAMT2like in roots. Each value represents the mean \pm SE $(n=3)$. Different lowercase letters indicate significant differences at $p<0.05$.

the expression of both genes, regardless of the organ examined, with the highest transcript amounts being observed in the afternoon/evening and the lowest in the morning (Figure 9A-D). In detail, BcAMT2 expression in leaves was lowest at 6:00 (only 10\% of that observed at 3:00) and highest at 18:00 (about 2-folds that observed at 3:00) (Figure 9A). Similarly, BcAMT2like transcripts reached the highest level at 18:00 and then decreased gradually to reach the lowest level at 9:00 (about 6\% of that observed at 3:00) (Figure 9B). In roots, the highest levels of BcAMT2 and BcAMT2like transcripts were observed at 24:00 (about 2.8-fold and 2.1-fold that observed at 3:00, respectively; Figure 9C, D). The expression of both $B c A M T 2 s$ in response to the circadian rhythm in roots occurred relatively later and for a shorter period than that in leaves.

\section{Discussion}

In the present study, analysis of cDNA and genomic DNA sequences of two members of the AMT2 subfamily from B. campestris, namely BcAMT2 and BcAMT2like, revealed that these genes encode polypeptides of 490 and 489 amino acids, respectively, and present $93 \%$ similarity at the amino acid level (Figure 1B). In agreement with the two to four introns structure presented by other AMT2 subfamily members (Castro-Rodríguez et al.
2016; Li et al. 2016; Sohlenkamp et al. 2002; Suenaga et al. 2003), BcAMT2 and BcAMT2like contain four introns (Figure $1 \mathrm{~A}$ ). The phylogenetic tree showed that BcAMT2 and BcAMT2like clustered within the AMT2 subfamily and were highly similar to BrAMT2s from $B$. rapa and AtAMT2 from A. thaliana (Figure 2A). Similar to other plant AMT proteins (Castro-Rodríguez et al. 2016; Thomas et al. 2000; von Wittgenstein et al. 2014), the deduced amino acid sequences of BcAMT2 and BcAMT2like contained 11 TM helices (Figure 1B), one extracellular N-terminus, and one cytosolic C-terminus, and had a potential AMT2 subfamily signature motif $\left({ }^{191}\right.$ DYSGGYVIHLSSGVAGFVAAYWVGPR $\left.{ }^{216}\right)$, located in the fifth transmembrane helix (Figures 1B, 2B). This motif, which is located in the most conserved TM (Saier 1999) can be used to identify additional members of the AMT subfamilies in other plant species (Couturier et al. 2007), and has been shown to affect ammonium transport capabilities of AMTs (Loqué et al. 2009; Wang et al. 2012). Similar to the results obtained for AMTs in other plants (Castro-Rodríguez et al. 2016; Sohlenkamp et al. 2002; Song et al. 2017; Yuan et al. 2009), both pBI121BcAMT2-GFP and pBI121-BcAMT2like-GFP localized to the plasma membrane. Furthermore, BcAMT2 and $B c A M T 2$ like could recover growth of mutant yeast cells when $2 \mathrm{mM}$ ammonium was the only $\mathrm{N}$ source (Figure 4).

Different members of the AMT family may have 
discrete or partially overlapping roles in plants (Couturier et al. 2007; Neuhäuser et al. 2009; Straub et al. 2014). The qPCR analysis showed that BcAMT2 and BcAMT2like were expressed constitutively throughout the plant and in all organs tested (Figure 5). Similar results have been obtained for LjAMT2;1 in L. japonicus (Simon-Rosin et al. 2003) and for PpAMT2;1 and PpAMT2;3 in Pinus pinaster (Castro-Rodríguez et al. 2016). Overall, BcAMT2 and $B c A M T 2 l i k e$ transcripts had similar expression tendencies from the cotyledon to the stalk-growth stage (Figure $5 \mathrm{~B}, \mathrm{C})$. While BcAMT2 was highly expressed in roots, leaves, flower buds, and pods (Figure 5B), BcAMT2like was mainly expressed in the vegetative tissues of fully developed plants and showed rather low expression in the reproductive tissues (Figure 5C). AMT2;1 plays an important role in root-to-shoot translocation of ammonium and is partly involved in ammonium uptake, depending on the plant nutritional status (Giehl et al. 2017). Whereas in the vegetative growth stages, both $B c A M T 2$ and BcAMT2like might transport ammonium from root to shoot, in the reproductive stage, $B c A M T 2$ might take up and transport ammonium to leaves, flower buds, and pods, while BcAMT2like might take up and transport ammonium to leaves.

Previous studies have shown that plant AMT genes can respond to various $\mathrm{N}$ regimes ( $\mathrm{Li}$ et al. 2016; Sohlenkamp et al. 2002; Song et al. 2017; Straub et al. 2014). In the current study, BcAMT2 expression was regulated by $\mathrm{N}$ starvation, ammonium supply (Figure 6), and high ammonium levels (Figure 7). Thus, BcAMT2s responded to various $\mathrm{N}$ regimes, and transcript levels in the leaves of $B$. campestris were positively correlated with the $\mathrm{N}$ concentration supplied to plants (Figures $6,7)$. During the $72 \mathrm{~h}$ of $\mathrm{N}$ starvation, BcAMT2 and $B c A M T 2$ like transcript levels in the leaves strongly declined, but under the $4 \mathrm{mM} \mathrm{NH}_{4} \mathrm{Cl}$ supply following $\mathrm{N}$ starvation, they returned to their basal levels (Figure $6 \mathrm{~A}, \mathrm{~B})$. Furthermore, their expression levels in leaves strongly increased under high ammonium treatment $\left(10 \mathrm{mM} \mathrm{NH}_{4} \mathrm{Cl}\right)$ (Li et al. 2016; Figure 7A, B). In roots, the transcript levels of $B c A M T 2 s$ responded differently to $\mathrm{N}$ starvation, ammonium supply (Figure $6 \mathrm{C}, \mathrm{D}$ ), and high ammonium levels (Figure 7C, D). Giehl et al. (2017) reported that AtAMT2;1 makes a small but important contribution to ammonium uptake in the millimolar range of substrate. In plants subjected to high ammonium concentration, AMT2;1 mediates a high accumulation ammonium in xylem sap, although no net ammonium influx, which contributes to long-distance translocation from root to shoot. These functions depended on organ-specific expression in response to the plant nutritional status (Couturier et al. 2007; Giehl et al. 2017; Li et al. 2016; Straub et al. 2014).

Owing to ammonia being a weak base, the balance between ammonia and ammonium depends on external
$\mathrm{pH}$. As $\mathrm{pH}$ increases from 5.5 to 7.0 , ammonium concentration decreases by about $1 \%$ while that of ammonia increases about 30-fold (Neuhäuser et al. 2009). Thus, profiles of $A M T$ responses to $\mathrm{pH}$ might be used to identify the substrate of AMT transportation as ammonia or ammonium (Li et al. 2016; Neuhäuser et al. 2009; Sohlenkamp et al. 2002). Compared with $\mathrm{pH}$ 4.8, BcAMT2 and BcAMT2like expression in leaves and roots decreased when plants were subjected to $\mathrm{pH} 5.8$, but increased significantly and returned to the original levels when subjected to $\mathrm{pH}$ 6.8. Ammonium transport is thought to be less affected by $\mathrm{pH}$ than that of ammonia (Sohlenkamp et al. 2002). Therefore, we hypothesized that the transportation abilities of BcAMT2s depend on external $\mathrm{pH}$. Both ammonium and ammonia might be transported by BcAMT2s in B. campestris, similar to observations in $A$. thaliana. Although ammonium is the recruited substrate of AtAMT2 (Sohlenkamp et al. 2002), the uncharged form ammonia is also transported (Neuhäuser et al. 2009). A mycorrhiza-specific LjAMT2;2 from $L$. japonicus can also transport electroneutral ammonia (Guether et al. 2009).

The circadian rhythm has been shown to regulate ammonium uptake in plants (Haydon et al. 2011), and there is substantial evidence indicating that the transcription of ammonium transporters is correlated with diurnal variation (Couturier et al. 2007; Gazzarrini et al. 1999; Li et al. 2016; Ranathunge et al. 2014; Song et al. 2011). In agreement with previous results (Couturier et al. 2007; Gazzarrini et al. 1999), BcAMT2 and BcAMT2like expression were strongly affected by diurnal changes, with the highest transcript levels in leaves being found at the end of the light period (Figure 9A, B), coinciding with the high carbohydrate levels required for ammonium assimilation (Couturier et al. 2007). This suggests that the regulation of transcription of $A M T$ genes might be controlled by carbohydrate availability (Couturier et al. 2007; Gazzarrini et al. 1999; Li et al. 2016). Likewise, BcAMT2s expression in the roots followed the circadian rhythm (Figure 9C, D); however, compared with leaves, the expression of $B c A M T 2 s$ in response to the circadian rhythm was comparatively delayed and occurred over a shorter period. These diurnal changes in AMT expression and transport activity have been attributed to changes in the concentration of photosynthetic products and their shoot-to-root transport (Camañes et al. 2007). Sugars make a major contribution to the diurnal changes in gene expression (Bläsing et al. 2005; Haydon et al. 2011). However, the effect of light stimulation on AMT expression and ammonium transport activity is independent of the circadian rhythm (Camañes et al. 2007). Therefore, further experiments will be necessary to elucidate the relationship between $B c A M T$ expression and light/dark period. 
In addition, ammonium in plant tissues is not only absorbed from the environment, but it is also produced in several metabolic processes, including nitrate reduction, photorespiration, amino acid catabolism, and phenylpropanoid metabolism (Bittsánszky et al. 2015; Liu and von Wirén 2017). Photorespiration in shoots generates a large amount of ammonium in mitochondria, which is transported to chloroplasts for re-assimilation and recycled to maintain N status (D'Apuzzo et al. 2004; Sohlenkamp et al. 2002). In addition, AMT expression is crucial to ensure ammonium recycling during photorespiration (D’Apuzzo et al. 2004; Li et al. 2016). Accordingly, we found that BcAMT2 expression in the leaves increased under high ammonium treatment (Figure 7). Thus, both BcAMT2 and BcAMT2like might participate in ammonium recycling during photorespiration (Li et al. 2016; Sohlenkamp et al. 2002).

BcAMT2 and BcAMT2like had similar responses to $\mathrm{N}$ regimen, external $\mathrm{pH}$, high ammonium treatment, and photoperiod, but also displayed specific expression patterns, with $B C A M T 2$ expression generally being higher than that of BcAMT2like. The BcAMT2s from B. campestris and BrAMT2s from B. rapa were located in the same phylogenetic cluster as AtAMT2 from A. thaliana (Figure 2A). However, the AMT2 subfamily in $A$. thaliana has only one member, while there are two members in B. campestris and B. rapa. Brassica plants have all undergone a whole-genome triplication event (Cheng et al. 2013) that might have originated the three paleo-subgenomes and multiple copies of paralogous genes found in the genomes of $B$. rapa and $B$. oleracea (Cheng et al. 2016), as observed for PtrAMT2;1 and PtrAMT2;2 (Couturier et al. 2007). Duplicate genes can potentially diverge in their roles as well as retain different sub-function of the original gene. Thus, the two $B$. campestris genes examined here might have developed sub-functions during evolution. Nevertheless, further experiments are required to assess the ability of ammonium absorption and/or transportation by BcAMT2 and BcAMT2like under different external pH.

\section{Acknowledgements}

This work was supported by the National Natural Science Foundation of China (31401855; http://www.nsfc.gov.cn/) and the China Agriculture Research System (CARS-25-C-04). The authors would also like to thank Dr. Bruno André (Université Libre de Bruxelles, Belgium) for providing the yeast mutant strain 31019b and Dr. Guibing Hu (South China Agricultural University, China) for providing $\mathrm{pBI} 121-\mathrm{GFP}$ vector.

\section{References}

Becker D, Stanke R, Fendrik I, Frommer WB, Vanderleyden J, Kaiser WM, Hedrich R (2002) Expression of the $\mathrm{NH}_{4}^{+}-$ transporter gene LeAMT1;2 is induced in tomato roots upon association with $\mathrm{N}_{2}$-fixing bacteria. Planta 215: 424-429
Bittsánszky A, Pilinszky K, Gyulai G, Komives T (2015) Overcoming ammonium toxicity. Plant Sci 231: 184-190

Bläsing OE, Gibon Y, Günther M, Höhne M, Morcuende R, Osuna D, Thimm O, Usadel B, Scheible WR, Stitt M (2005) Sugars and circadian regulation make major contributions to the global regulation of diurnal gene expression in Arabidopsis. Plant Cell 17: 3257-3281

Bloom AJ, Meyerhoff PA, Taylor AR, Rost TL (2002) Root development and absorption of ammonium and nitrate from the rhizosphere. J Plant Growth Regul 21: 416-431

Camañes G, Cerezo M, Primo-Millo E, Gojon A, García-Agustín P (2007) Ammonium transport and CitAMT1 expression are regulated by light and sucrose in Citrus plants. J Exp Bot 58: 2811-2825

Camañes G, Cerezo M, Primo-Millo E, Gojon A, García-Agustín P (2009) Ammonium transport and CitAMT1 expression are regulated by $\mathrm{N}$ in Citrus plants. Planta 229: 331-342

Castro-Rodríguez V, Assaf-Casals I, Pérez-Tienda J, Fan XR, Avila C, Miller A, Cánovas FM (2016) Deciphering the molecular basis of ammonium uptake and transport in maritime pine. Plant Cell Environ 39: 1669-1682

Cheng F, Mandáková T, Wu J, Xie Q, Lysak MA, Wang XW (2013) Deciphering the diploid ancestral genome of the mesohexaploid Brassica rapa. Plant Cell 25: 1541-1554

Cheng F, Sun RF, Hou XL, Zheng HK, Zhang FL, Zhang YY, Liu B, Liang JL, Zhuang M, Liu YX, et al. (2016) Subgenome parallel selection is associated with morphotype diversification and convergent crop domestication in Brassica rapa and Brassica oleracea. Nat Genet 48: 1218-1224

Couturier J, Montanini B, Martin F, Brun A, Blaudez D, Chalot M (2007) The expanded family of ammonium transporters in the perennial poplar plant. New Phytol 174: 137-150

Crooks GE, Hon G, Chandonia J, Brenner SE (2004) WebLogo: A Sequence Logo Generator. Genome Res 14: 1188-1190

D’Apuzzo E, Rogato A, Simon-Rosin U, El Alaoui H, Barbulova A, Betti M, Dimou M, Katinakis P, Marquez A, Marini AM, et al. (2004) Characterization of three functional high-affinity ammonium transporters in Lotus japonicus with differential transcriptional regulation spatial expression. Plant Physiol 134: 1763-1774

Ding L, Li YR, Wang Y, Gao LM, Wang M, Chaumont F, Shen QR, Guo SW (2016) Root ABA accumulation enhances rice seedling drought tolerance under ammonium supply: Interaction with aquaporins. Front Plant Sci 7: 1206

Fernández-Crespo E, Scalschi L, Llorens E, García-Agustín P, Camañes $\mathrm{G}$ (2015) $\mathrm{NH}_{4}^{+}$protects tomato plants against Pseudomonas syringae by activation of systemic acquired acclimation. J Exp Bot 66: 6777-6790

Gazzarrini S, Lejay L, Gojon A, Ninnemann O, Frommer WB, vonWirén N (1999) Three functional transporters for constitutive, diurnally regulated, and starvation-induced uptake of ammonium into Arabidopsis roots. Plant Cell 11: 937-947

Giehl RFH, Laginha AM, Duan FY, Rentsch D, Yuan LX, von Wirén N (2017) A critical role of AMT2;1 in root-to-shoot translocation of ammonium in Arabidopsis. Mol Plant 10: 1449-1460

Guether M, Neuhauser B, Balestrini R, Dynowski M, Ludewig U, Bonfante P (2009) A mycorrhizal-specific ammonium transporter from Lotus Japonicus acquires nitrogen released by Arbuscular Mycorrhizal Fungi. Plant Physiol 150: 73-83

Hachiya T, Sakakibara H (2017) Interactions between nitrate and ammonium in their uptake, allocation, assimilation, and signaling in plants. J Exp Bot 68: 2501-2512

Hao DL, Yang SY, Huang YN, Su YH (2016) Identification of 
structural elements involved in fine-tuning of the transport activity of the rice ammonium transporter OsAMT1;3. Plant Physiol Biochem 108: 99-108

Haydon MJ, Bell LJ, Webb AAR (2011) Interactions between plant circadian clocks and solute transport. J Exp Bot 62: 2333-2348

Hu B, Jin JP, Guo AY, Zhang H, Luo JC, Gao G (2015) GSDS 2.0: An upgraded gene feature visualization server. Bioinformatics 31 : 1296-1297

Jing J, Zhang F, Rengel Z, Shen J (2012) Localized fertilization with $\mathrm{P}$ plus $\mathrm{N}$ elicits an ammonium-dependent enhancement of maize root growth and nutrient uptake. Field Crop Res 133: 176-185

Li H, Cong Y, Chang YH, Lin J (2016) Two AMT2-type ammonium transporters from pyrus betulaefolia demonstrate distinct expression characteristics. Plant Mol Biol Rep 34: 707-719

Lima JE, Kojima S, Takahashi H, von Wirén N (2010) Ammonium triggers lateral root branching in Arabidopsis in an ammonium transporter1;3-dependent manner. Plant Cell 22: 3621-3633

Liu Y, von Wirén N (2017) Ammonium as a signal for physiological and morphological responses in plants. J Exp Bot 68: 2581-2592

Livak KJ, Schmittgen TD (2001) Analysis of relative gene expression data using real-time quantitative PCR and the $2^{-\Delta \Delta \mathrm{Ct}}$ method. Methods 25: 402-408

Loqué D, Mora SI, Andrade SL, Pantoja O, Frommer WB (2009) Pore mutations in ammonium transporter AMT1 with increased electrogenic ammonium transport activity. J Biol Chem 284: 24988-24995

Loqué D, von Wirén N (2004) Regulatory levels for the transport of ammonium in plant roots. J Exp Bot 55: 1293-1305

Ludewig U, Neuhäuser B, Dynowski M (2007) Molecular mechanisms of ammonium transport and accumulation in plants. FEBS Lett 581: 2301-2308

Marini AM, Soussi-Boudekou S, Vissers S, Andre B (1997) A family of ammonium transporters in Saccharomyces cerevisiae. Mol Cell Biol 17: 4282-4293

McDonald TR, Dietrich FS, Lutzoni F (2012) Multiple horizontal gene transfers of ammonium transporters/ammonia permeases from prokaryotes to eukaryotes: Toward a new functional and evolutionary classification. Mol Biol Evol 29: 51-60

Neuhäuser B, Dynowski M, Ludewig U (2009) Channel-like $\mathrm{NH}_{3}$ flux by ammonium transporter AtAMT2. FEBS Lett 583: 2833-2838

Pantoja O (2012) High affinity ammonium transporters: Molecular mechanism of action. Front Plant Sci 3: 34

Ranathunge K, El-kereamy A, Gidda S, Bi YM, Rothstein SJ (2014) AMT1;1 transgenic rice plants with enhanced $\mathrm{NH}_{4}^{+}$permeability show superior growth and higher yield under optimal and suboptimal $\mathrm{NH}_{4}^{+}$conditions. J Exp Bot 65: 965-979

Saier MH Jr (1999) Genome archeology leading to the characterization and classification of transport proteins. Curr Opin Microbiol 2: 555-561

Salvemini F, Marini AM, Riccio A, Patriarca EJ, Chiurazzi M (2001) Functional characterization of an ammonium transporter gene from Lotus japonicus. Gene 270: 237-243

Simon-Rosin U, Wood C, Udvardi MK (2003) Molecular and cellular characterisation of $L j A M T 2 ; 1$, an ammonium transporter from the model legume Lotus japonicus. Plant Mol Biol 51: 99-108

Sohlenkamp C, Wood CC, Roeb GW, Udvardi MK (2002) Characterization of Arabidopsis AtAMT2, a high-affinity ammonium transporter of the plasma membrane. Plant Physiol 130: $1788-1796$

Song SW, He ZH, Huang XM, Zhong LH, Liu HC, Sun GW, Chen
RY (2017) Cloning and characterization of the ammonium transporter genes $B a A M T 1 ; 1$ and BaAMT1;3 from Chinese kale. Hortic Environ Biote 58: 178-186

Song SW, Yi LY, Liu HC, Sun GW, Chen RY (2012) Effect of ammonium and nitrate ratio on nutritional quality of flowering Chinese cabbage. Appl Mech Mater 142: 188-192

Song T, Gao Q, Xu Z, Song RT (2011) The cloning and characterization of two ammonium transporters in the saltresistant green alga, Dunaliella viridis. Mol Biol Rep 38: 4797-4804

Straub D, Ludewig U, Neuhäuser B (2014) A nitrogen-dependent switch in the high affinity ammonium transport in Medicago truncatula. Plant Mol Biol 86: 485-494

Suenaga A, Moriya K, Sonoda Y, Ikeda A, von Wirén N, Hayakawa T, Yamaguchi J, Yamaya T (2003) Constitutive expression of a novel type ammonium transporter OsAMT2 in rice plants. Plant Cell Physiol 44: 206-211

Tamura K, Stecher G, Peterson D, Filipski A, Kumar S (2013) MEGA6: Molecular evolutionary genetics analysis version 6.0. Mol Biol Evol 30: 2725-2729

Thomas GH, Mullins JG, Merrick M (2000) Membrane topology of the Mep/Amt family of ammonium transporters. Mol Microbiol 37: 331-344

von Wirén N, Gazzarrini S, Gojon A, Frommer WB (2000a) The molecular physiology of ammonium uptake and retrieval. Curr Opin Plant Biol 3: 254-261

von Wirén N, Lauter FR, Ninnemann O, Gillissen B, Walch-Liu P, Engels C, Jost W, Frommer WB (2000b) Differential regulation of three functional ammonium transporter genes by nitrogen in root hairs and by light in leaves of tomato. Plant J 21: 167-175

von Wittgenstein NJ, Le CH, Hawkins BJ, Ehlting J (2014) Evolutionary classification of ammonium, nitrate, and peptide transporters in land plants. BMC Evol Biol 14: 11

Wang S, Orabi EA, Baday S, Berneche S, Lamoureux G (2012) Ammonium transporters achieve charge transfer by fragmenting their substrate. J Am Chem Soc 134: 10419-10427

Yang N, Zhu CH, Gan LJ, Ng D, Xia K (2011) Ammoniumstimulated root hair branching is enhanced by methyl jasmonate and suppressed by ethylene in Arabidopsis thaliana. J Plant Biol 54: 92-100

Yuan LX, Graff L, Loqué D, Kojima S, Tsuchiya YN, Takahashi H, von Wiren N (2009) AtAMT1;4, a pollen-specific high-affinity ammonium transporter of the plasma membrane in Arabidopsis. Plant Cell Physiol 50: 13-25

Yuan LX, Loqué D, Kojima S, Rauch S, Ishiyama K, Inoue E, Takahashi H, von Wirén N (2007) The organization of highaffinity ammonium uptake in Arabidopsis roots depends on the spatial arrangement and biochemical properties of AMT1-type transporters. Plant Cell 19: 2636-2652

Zeng HQ, Liu G, Kinoshita T, Zhang RP, Zhu YY, Shen QR, Xu GH (2012) Stimulation of phosphorus uptake by ammonium nutrition involves plasma membrane $\mathrm{H}^{+}$-ATPase in rice roots. Plant Soil 357: 205-214

Zhang R, Zhu J, Cao HZ, An YR, Huang JJ, Chen XH, Mohammed N, Afrin S, Luo ZY (2013) Molecular cloning and expression analysis of PDR1-like gene in ginseng subjected to salt and cold stresses or hormonal treatment. Plant Physiol Biochem 71: 203-211

Zou C, Shen J, Zhang F, Guo S, Rengel Z, Tang C (2001) Impact of nitrogen form on iron uptake and distribution in maize seedlings in solution culture. Plant Soil 235: 143-149 